\title{
Optimum Functions for Radial Wires of Monopole Antennas with Arbitrary Elevation Angles
}

\author{
Mahdi FARTOOKZADEH ${ }^{1}$, Seyyed Hossein MOHSENI ARMAKI ${ }^{1}$, \\ Seyyed Mohamad Javad RAZAVI ${ }^{1}$, Jalil RASHED-MOHASSEL ${ }^{2}$ \\ ${ }^{1}$ Dept. of Electrical and Electronics Engineering, Malek Ashtar University, P. O. Box 1774-15875, Tehran, Iran \\ ${ }^{2}$ Center of Excellence on Applied Electromagnetic Systems, School of ECE, College of Engineering, Univ. of Tehran, \\ P. O. Box 14395-515, Tehran, Iran
}

mahdi.fartookzadeh@gmail.com,mohseni@ee.iust.ac.ir, razavismj@yahoo.com, jrashed@ut.ac.ir

Manuscript received August 1, 2015

\begin{abstract}
Monopole antennas on the earth usually use ground screen with simple radial wires to improve their radiation performance. The number of radials $N$ is usually considered a constant in the screen. This paper studies the effect of changing $N$ and considering it as a function of distance $\rho$ from the monopole using a simple and yet a fast method. The function $N(\rho)$ is optimized for different beam angles of an HF monopole antenna. The theoretical functions are converted to practical functions to be formed using meandered lines. Practicable calculated results are validated by method of moments. Furthermore it is shown that for low angle radiation a constant $N(\rho)$ with optimized radius of the ground screen is the best choice. The results can be used for higher frequencies, i.e. VHF and UHF frequency bands as well.
\end{abstract}

\section{Keywords}

Compensation theorem, antenna ground plane, antenna impedance, HF propagation, monopole antenna, radial wires

\section{Introduction}

A classic area of interest in radio wave propagation is the monopole antenna over a lossy ground at LF, MF and HF bands [1]-[3]. For example for the miniaturization of these antennas several structures have been introduced such as top-loaded structures, optimized wire structures, etc. [4]-[8]. An important task for the improvement of antenna characteristics is the use of radial wires grid as the ground [9]-[12]. Calculation of the radiated fields of a monopole on a homogeneous ground is simplified using Sommerfeld integrals in early twentieth. Furthermore, developments on the applied Schelkunoff's equivalence theorem and compensation theorem (based on Lorentz reciprocity) arose, simultaneously and after a period of time. Subsequently, the effect of ground screen on the impedance calculation and radiation pattern was studied extensively. The most frequent method was the usage of compensation theorem that was studied by Wait and others in about a quarter of century [2], [11]. Some other approaches are used for this purpose such as equivalence theorem [13] and extinction theorem [14]. A comparatively new approach is the method of moments (MoM) which is outspreaded by the enhancement of computers [15]-[17]. Nevertheless, there are still some limitations for this method. As an example for the optimization of large structures the required time by this method may be considerable. Therefore, the previously introduced analytical methods or the combination of the analytical and numerical methods may provide the best solution for optimization and the results can be validated using new numerical codes. An interesting conversation about the comparison between this method and previous methods can be found in [18].

The use of a radial wire grid is a special kind of ground screens that is assumed to have identical impedance in $\phi$ direction of cylindrical coordinates [2], [19], [20]. The number of wires is usually in order of 100 and does not change in $\hat{\rho}$ direction (Fig. 1). A financial approach to the optimization of the number of radial wires is introduced in the past [21] with constant number of radials. This number is considered constant because of two reasons. First, if the total current is considered $I_{0}$ the current distribution will be $J_{\mathrm{S}}(\rho)=I_{0} /(2 \pi \rho)$ and this current is identical to the conductor density for constant number of radials through $\rho$. It is observed that, this is not an accurate conclusion, since the effect of ground loss, reflections and wires radiation are neglected. Second, implementation of varying $N$ is difficult and in some cases impossible. This will be resolved using meandered lines.

This paper studies the effects of changes on the number of radial wires in $\rho$ direction and looks for a function that produces a radiation pattern with a determined beam elevation angle for a quarter wave monopole antenna. The proposed ground screens may be used for HF, VHF, UHF and higher frequencies. HF band is selected to indicate the results, since it has low and high angle applications and similar results may be obtained for higher frequencies by scaling the structures. 
It will be seen that the effect of this change in the number of wires is very significant on the radiation pattern of a monopole antenna for large radius screens. This approach is uninhibited to study for a discrete function as can be seen at the end of [13] and [14]. Therefore, the number of radial wires is shown by $N(\rho)$ and this positive function is to be optimized. It is considered that the total length of the wires is constant and equal to $l_{\mathrm{gs}}$ and the radius of the ground screen is also constant and equals $a$. The obtained function may be hypothetical for real antennas but gives good understanding of the effect of ground screen impedance. The next step is to use appropriate limits to make the function more practical and construct the function by meandered lines.

\section{Mathematical and Physical Background}

\subsection{Background Formulation}

The impedance of a ground system with $N$ radial wires can be obtained using an approximately equivalent system, wire grid with the separation $d=2 \pi \rho / N$ [2]. The schematics of wire grid and radial wires are indicated in Fig. 1 (a) and (b), respectively. A key equation to provide the equivalent impedance for the ground system with wire radius $R_{\mathrm{w}}$ and distance $d$ or total number $N$,

$$
Z_{\mathrm{s}}(\rho)=\left(\frac{\mathrm{j} \omega \mu_{0} d}{2 \pi}\right) \ln \left(\frac{d}{2 \pi R_{\mathrm{w}}}\right)=\left(\frac{\mathrm{j} \omega \mu_{0} \rho}{N}\right) \ln \left(\frac{\rho}{N R_{\mathrm{w}}}\right)
$$

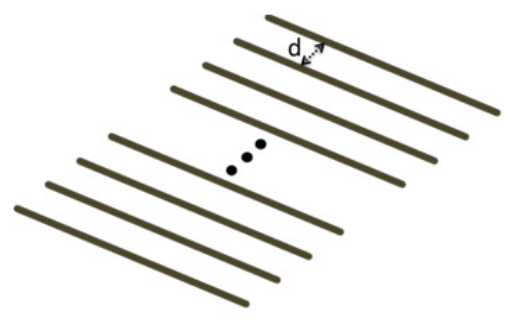

(a) is introduced by Wessel [21] and generalized by Macfarlane [22]. This impedance is considered parallel to the ground impedance,

$$
Z(\theta)=\sqrt{\frac{\mathrm{j} \mu_{0} \omega}{\sigma+\mathrm{j} \varepsilon \omega}} \sqrt{1-\frac{\mathrm{j} \varepsilon_{0} \omega}{\sigma+\mathrm{j} \varepsilon \omega} \sin ^{2} \theta},
$$

that is a function of angle as indicated in Fig. 1(c). Consequently, the total impedance

$$
Z^{\prime}(\theta, \rho)=\frac{Z(\theta) Z_{\mathrm{s}}(\rho)}{Z(\theta)+Z_{\mathrm{s}}(\rho)}
$$

is a function of $\theta$ and $\rho$. The ground loss is shown by $\sigma$ in (2) which is usually used for a conductive material. Anyway we observe in some references that a complex number is used for $\varepsilon_{\mathrm{r}}=\varepsilon_{\mathrm{r}}^{\prime}-\mathrm{j} \varepsilon_{\mathrm{r}}^{\prime \prime}$. It can be obtained from $\sigma$ explicitly as

$$
\varepsilon^{\prime \prime}=\frac{\sigma}{2 \pi f} \Rightarrow \varepsilon_{\mathrm{r}}^{\prime \prime}=\frac{\sigma}{2 \pi f \varepsilon_{0}} .
$$

Now if we indicate the far-fields in the absence of the screen by $F_{0}(\theta)$, by multiplying the correction function on $F_{0}(\theta)$, the far-fields with the screen for a quarter wave monopole will be calculated as [2],

$$
\begin{aligned}
& F_{\mathrm{CN}}(\theta)=F_{0}(\theta) \\
& {\left[\begin{array}{l}
1-\frac{k \sin \theta}{\cos \left(\frac{\pi}{2} \cos \theta\right)} \cdot \int_{0}^{a} W^{\prime}(\theta, k \rho) \frac{Z(\theta)-Z^{\prime}(\theta, \rho)}{\eta_{0}} e^{\left.-\mathrm{j} k \sqrt{\rho^{2}+(\lambda / 4)}\right)^{2}} \\
\cdot J_{1}(k \rho \sin \theta) \mathrm{d} \rho
\end{array}\right]}
\end{aligned}
$$

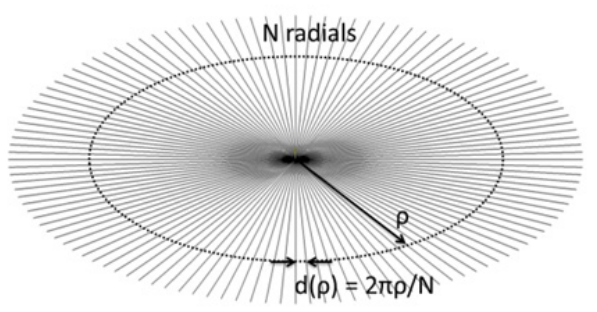

(b)

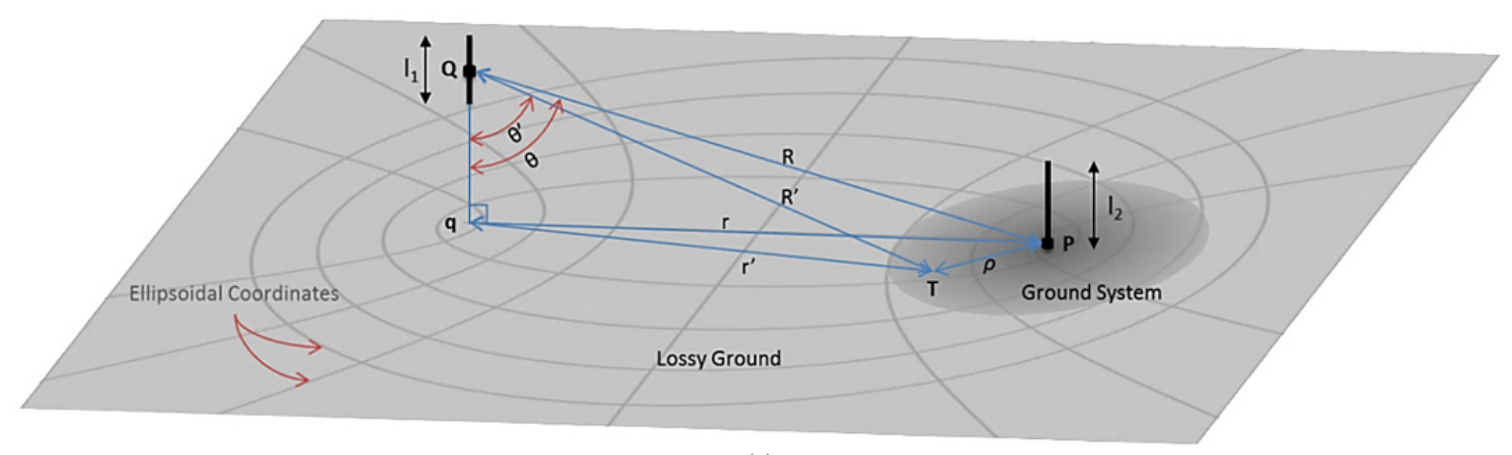

(c)

Fig. 1. Schematics of (a) wire grid and (b) radial wires for impedance calculation, (c) the antenna with ground system (P) and the antenna (Q) to be used for compensation theorem. 
where $W^{\prime}(\theta, k \rho)$ can be obtained using compensation theorem and numerical methods. As indicated in Fig. 1(c) a dipole is considered at the considerable distance $R$ from the monopole antenna with the ground screen of area $S$. Consequently, the difference between the impedances are obtained using

$$
\Delta Z=Z_{\mathrm{q}}-Z_{\mathrm{p}}=\frac{1}{I_{0}^{2}} \iint_{S}\left(Z^{\prime}-Z\right) H_{\mathrm{pt}} \cdot H_{\mathrm{qt}} \mathrm{d} S .
$$

Mutual impedance for the first antenna is known and is given by [2]

$$
Z_{\mathrm{q}}=\frac{\mathrm{j} \mu_{0} \omega l_{1} l_{2}}{2 \pi R} e^{-\mathrm{j} k R} \sin ^{2} \theta W(r, Z) .
$$

For the second antenna, the mutual impedance is therefore considered to have the form

$$
Z_{\mathrm{p}}=\frac{\mathrm{j} \mu_{0} \omega l_{1} l_{2}}{2 \pi R} e^{-j k R} \sin ^{2} \theta W^{\prime}\left(r, Z, Z^{\prime}\right),
$$

with an unknown $W^{\prime}$. Also for a point on the ground surface with distances $R^{\prime}$ and $\rho$ from the first and second antennas respectively, with $k R^{\prime}>>1$ and $\left|Z / \eta_{0}\right|^{2}<<1$, we have

$$
H_{\mathrm{qt}}=\frac{\mathrm{j} k I_{0} l_{1}}{2 \pi R^{\prime}} e^{-\mathrm{j} k R^{\prime}} \sin \theta^{\prime} W\left(r^{\prime}, Z\right)
$$

and we can conclude for the second antenna (note that we do not have $k \rho>>1$ )

$$
H_{\mathrm{pt}}=\frac{\mathrm{j} k I_{0} l_{2}}{2 \pi \rho}\left(1+\frac{1}{\mathrm{j} k \rho}\right) e^{-\mathrm{j} k \rho} W^{\prime}\left(\rho, Z, Z^{\prime}\right) .
$$

An equation with a surface integral will be obtained by substituting (7) and (8) in (6). A classic problem which can be found in several reports such as [23] is to form a standard integral equation using ellipsoidal coordinates [24] and the stationary phase method [25]. Anyway, with the assumption of a symmetric ground screen, it produces a Volterra integral equation of the second kind [2]

$$
\begin{aligned}
& W^{\prime}(\theta, \rho)=W(\theta, \rho)+ \\
& \left(\frac{\mathrm{j} \rho}{2 \pi}\right)^{\frac{1}{2}} \int_{0}^{\rho} \frac{Z(\theta)-Z^{\prime}\left(\theta, \rho^{\prime}\right)}{\eta_{0} \sqrt{\rho^{\prime}\left(\rho-\rho^{\prime}\right)}} \cdot W\left(\theta, \rho-\rho^{\prime}\right) W^{\prime}\left(\theta, \rho^{\prime}\right) \mathrm{d} \rho^{\prime}
\end{aligned}
$$

where

$$
W(\theta, x)=1-\mathrm{j} \sqrt{\pi p} e^{-p} \operatorname{erfc}(\mathrm{j} \sqrt{p}), \quad p=-\left(\frac{\mathrm{j} x}{2}\right)\left[\frac{Z(\theta)}{\eta_{0}}\right]^{2}
$$

and the detailed derivation is provided by Green in [23]. $W^{\prime}(\theta, \rho)$ can be considered unity for small screens on high lossy ground, however should be solved for general screens. Now this is not a problematic task, while many numerical methods are available. Therefore, $W^{\prime}(\theta, \rho)$ is calculated for three kinds of grounds with poor, normal and high conductivity in Fig. 2(a) to Fig. 2(c). $W^{\prime}(\theta, \rho)$ is closer to 1 when the conductivity of ground increases. In addition, it can be observed that for high conductive grounds the dependency of $W^{\prime}(\theta, \rho)$ on $\theta$ is reduced. Therefore the effect of ground screen or radial wires is negligible for high conductive grounds.

Finally, $F_{0}(\theta)$ can be replaced by either of $E_{0}(\theta)$ and $H_{0}(\theta)$. Norton separated the far-field and indicated that for large distances we have [3]

$$
E_{0}(\theta)=-\hat{\theta} \frac{\mathrm{j} \eta_{0} k I l}{4 \pi r_{1}} e^{-\mathrm{j} k r_{1}}\left(1+R_{v}\right) \sin \theta
$$

and

$$
H_{0}(\theta)=-\hat{\phi} \frac{\mathrm{j} k I l}{4 \pi r_{1}} e^{-\mathrm{j} k r_{1}}\left(1+R_{v}\right) \sin \theta
$$

where

$$
R_{v}=\frac{\cos \theta-Z / \eta_{0}}{\cos \theta+Z / \eta_{0}}
$$

and $r_{1}$ is the distance of observation point from the antenna.

\subsection{Physical Background}

The best ground for low angle radiation of a monopole antenna is an infinite size PEC plane that produces the maximum directivity of $5.17 \mathrm{~dB}$ at $\theta=90^{\circ}$. However, ground plane size is limited in practice and therefore the beam angle rises as indicated in Fig. 3(a) for a ground plane with a radius of say $5 \lambda$. The frequency is $10 \mathrm{MHz}$, and the ground has the conductivity $\sigma=0.003 \mathrm{~S} / \mathrm{m}$ for all calculations and simulations of the paper from now on. Therefore the height of the monopole is $7.5 \mathrm{~m}$ and the radius of the ground screen or the radial wires' lengths are $150 \mathrm{~m}$. The impedance of a PEC plane is zero and it makes $Z^{\prime}$ zero in (5) and therefore this is the best situation for low angle radiation (large $\theta \mathrm{s}$ ). Nevertheless, in many practical situations a metallic plane cannot be used for the antenna especially for large size screens. The metallic plane can be replaced by radial wires for large size ground screens as indicated in Fig. 3(b).

Radiation pattern of a quarter wave monopole with 150 radials of $5 \lambda$ long is shown in Fig. 3(c) as an example. The calculated (Cal.) results are obtained from the introduced method in (5) and the MoM of FEKO has been used for simulation (Sim.). Some oscillations with five concave parts are observed in the calculated and simulated radiation patterns. It can be shown that the number of concave parts is dependent on the length of radials $a$ in $\lambda$. The only part in (5) that has a dependency on $a$ is the integral with the limits 0 and $a$. In addition, we do not observe any oscillations in $W^{\prime}$ curves in Fig. 2. Therefore the sources of these oscillations in (5) can be $Z^{\prime}(\theta, \rho)$ and $J_{1}(k \rho \sin \theta)$ those are dependent on both of $\theta$ and $\rho$. However for a constant $N$, $Z^{\prime}(\theta, \rho)$ cannot produce oscillations since the only dependency of $Z^{\prime}(\theta, \rho)$ on $\rho$ is in (1) which is not an oscillating function. Anyway, it will be observed that $Z^{\prime}(\theta, \rho)$ may have some effects on the oscillations when $N$ is considered as the function of $\rho$. In this situation $Z^{\prime}(\theta, \rho)$ is not obtained from (3) explicitly and will be obtained using the 


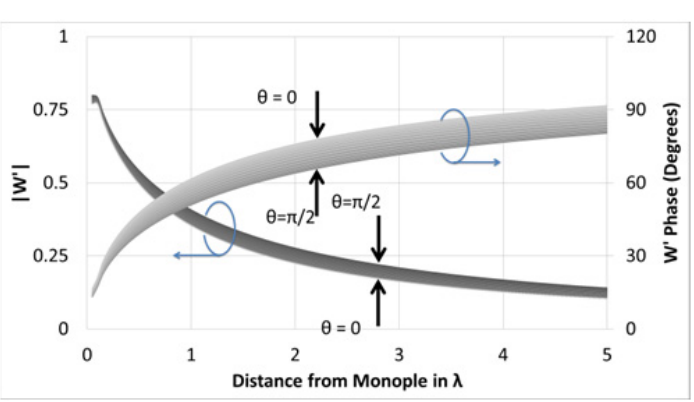

(a)

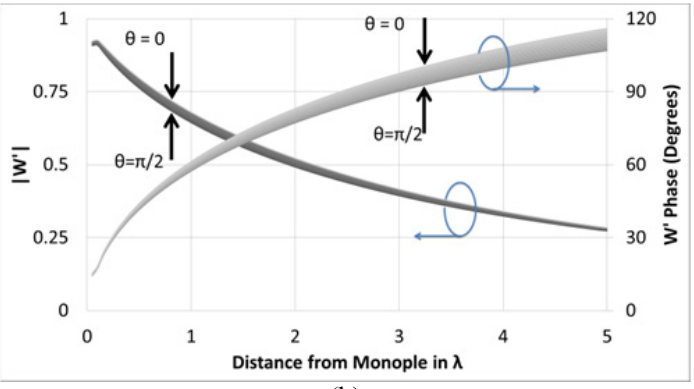

(b)

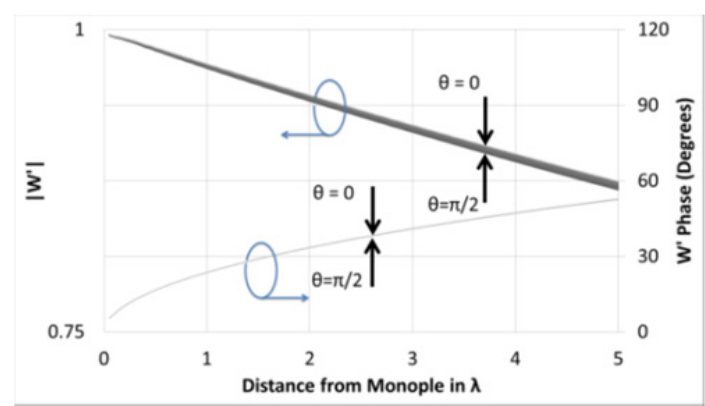

(c)

Fig. 2. $W^{\prime}$ of a $150 \mathrm{~m}$ ground screen at $10 \mathrm{MHz}$ with $N=150$ and $R_{\mathrm{w}}=5 \mathrm{~mm}$ on a lossy ground with (a) $\sigma=$ $0.0003 \mathrm{~S} / \mathrm{m}$, (b) $\sigma=0.003 \mathrm{~S} / \mathrm{m}$ and (c) $\sigma=0.03 \mathrm{~S} / \mathrm{m}$.

equivalency principle as will be discussed shortly. Consequently, radiation pattern will be a complicated function of $N(\rho)$ and $\theta$ which can be modified by varying $N(\rho)$ and can be maximized at a given $\theta$. The fields at the given $\theta$ is actually obtained by superposition of the fields from wires at all distances, $\rho$ s, from the antenna. Note that changing $N(\rho)$ can produce maximums at desired elevation angles since it will appear with different phases, $k \sqrt{\rho^{2}+(\lambda / 4)^{2}}$, at different distances $\rho$ in the integral, (5).

\section{Finding the Optimum Function}

We would like to change the numbers of radials theoretically and observe the resulting radiation pattern. An immediate method is to consider $N$ as a function of $\rho$ and substitute the constant $N$ in (1), (5) and the integral equation in (7). This is not an accurate method however. A better approach is to calculate the fields of each radius $\rho$ by it's $N(\rho)$ and subtract it by the calculated fields of $\rho-\mathrm{d} \rho$ with $N(\rho)$ and integrate from zero to $a$

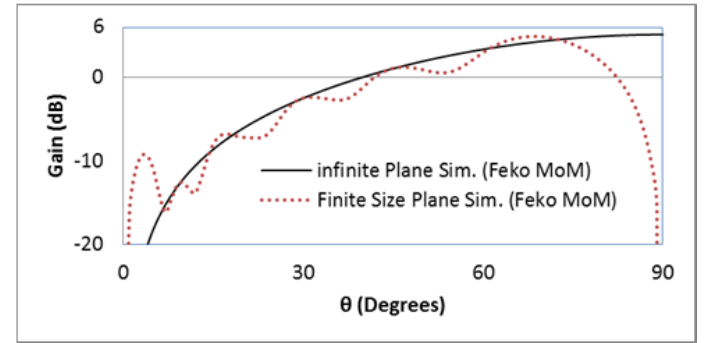

(a)

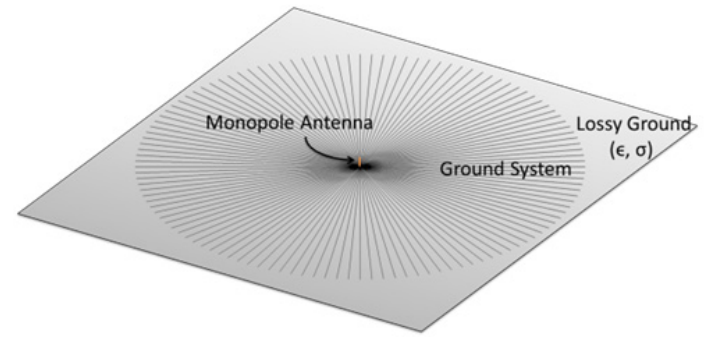

(b)

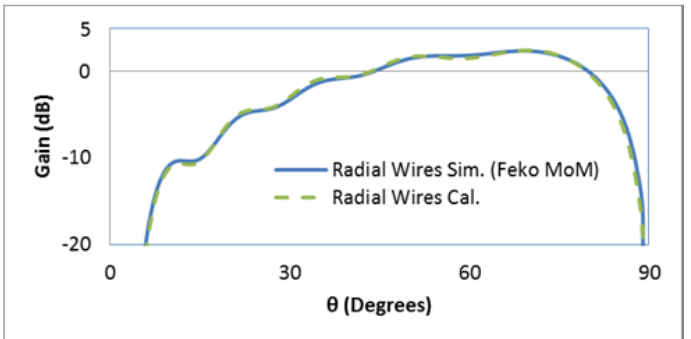

(c)

Fig. 3. A monopole antenna on the ground; (a) radiation pattern of a $\lambda / 4$ monopole on the infinite PEC plane and on a PEC plane with $5 \lambda$ radius over the normal lossy ground , (b) the schematic and (c) calculated and simulated radiation patterns of a $\lambda / 4$ monopole with 150 radial wires. (Conductivity of the normal ground is $\sigma=0.003 \mathrm{~S} / \mathrm{m})$.

$$
F_{\mathbf{V N}}=\int_{0}^{a_{t}} \frac{\partial F_{\mathbf{C N}}\left(N\left(\rho=\rho^{\prime}\right), a=\rho^{\prime}\right)}{\partial a} \mathrm{~d} \rho^{\prime}
$$

where $F_{\mathrm{CN}}$ is the field of an antenna with constant number of radials with a given $N$ and $a$ and $F_{\mathrm{VN}}$ is the field of the antenna with variable number of radials $N(\rho)$.

The function $F_{\mathrm{VN}}$ does not have a general closed form solution and involves an integral equation that has to be solved numerically. Therefore, for the calculation of this integral it should be discretized. For each section, two integral equations should be solved. In the discrete form it will be

$$
\begin{aligned}
F_{\mathbf{V N}}\left(N(\rho), a_{t}\right)= & \\
& F_{\mathbf{C N}}\left(N\left(\rho=\Delta \rho^{\prime}\right), a=\Delta \rho^{\prime}\right)+ \\
& \frac{a_{t}}{\Delta \rho^{\prime}}-1 \\
\frac{\rho^{\prime}}{\Delta \rho^{\prime}}=1 & {\left[\begin{array}{l}
F_{\mathbf{C N}}\left(N\left(\rho=\rho^{\prime}\right), a=\rho^{\prime}+\Delta \rho^{\prime}\right)- \\
F_{\mathbf{C N}}\left(N\left(\rho=\rho^{\prime}\right), a=\rho^{\prime}\right)
\end{array}\right] . }
\end{aligned}
$$

Now $F_{\mathrm{VN}}$ can be obtained for an arbitrary $N(\rho)$. However, there are some limitations for this function, 
which can be formulated as

$$
\left\{\begin{array}{c}
N(\rho)>N_{\text {min }} \\
N(\rho)<N_{\max } \\
l_{\mathrm{gs}}=\int_{0}^{a} N(\rho) \mathrm{d} \rho
\end{array}\right.
$$

For example for the constant $N(\rho)$, it should be equal to $l_{\mathrm{gs}} / a$. Also $N_{\min }$ should be sufficient to keep the angle between wires low and preserve the accuracy of (1). Here we assume $N_{\min }=60$ (which leads to $6^{\circ}$ difference between neighboring wires), $N_{\max }=500$ and (12) has 50 sections. Now one can establish an optimization with the components of $N(\rho)$ as the variables and the goal function $\left.F_{\mathrm{VN}}\right|_{\theta=\theta_{\text {req }}}$ to be maximized at $\theta_{\text {req }}$.

Consequently, the schematic of ground screen with optimum number of radials with $5 \lambda$ screen radius for the maximum beam angle $(\theta)$ of say $45^{\circ}$ is illustrated in Fig. 4. It is clear that this schematic is hypothetical since the wires are not connected. A practical structure will be introduced in the next section. The obtained function in Fig. 4 is almost independent from the characteristics of the ground, because as explained in the second section the fields are calculated using compensation theorem and the impedance of the screen is considered parallel to the impedance of the ground. Therefore, the results can be similar for other kinds of grounds such as poor or high conductive grounds. In addition, we are not required to assume a frequency for our calculations if the dimensions are given in terms of $\lambda$ and $\varepsilon^{\prime \prime}$ is used instead of $\sigma$. Therefore the results may be applied for all frequencies. However if we use $\sigma$, it has to be scaled with frequency to produce similar results at all frequencies as can be observed in (2) and (4).

\section{Practical Modification and Results}

It is clear that changing $N(\rho)$ as the calculated function in previous section is not practical. Therefore, in this section we introduce a practical solution for this function that meets approximately the advantages of previously introduced functions. For example we can choose the function as

$$
N(\rho)=\left\{\begin{array}{cc}
90, & 0<\rho<l_{1}-l_{\mathrm{a}} \\
180, & l_{1}-l_{\mathrm{a}}<\rho<l_{1} \\
90, & l_{1}<\rho<150 \mathrm{~m}
\end{array}\right.
$$

The schematic of radial wires and the practical substitution is shown in Fig. 5. In the practical structure the open wires are put in the circuit as the meandered lines. Therefore we should have $l_{\mathrm{a}} \cong l_{\mathrm{m}}$ and $l_{\mathrm{m}}=n \lambda, n=1,2,3, \ldots$ in Fig. 5 to meet the boundary condition for the currents at point A. Some examples of the calculated and simulated radiation patterns of a $\lambda / 4$ antenna on the specified ground screen with $l_{\mathrm{m}}=\lambda$ on a normal ground is given in Fig. 6 . It can be observed that changing $l_{1}$ in (14) affects the oscillations of the radiation pattern. Now by controlling these oscillations one may obtain the radiation pattern with the desired angle of maximum directivity. In addition, it can be seen that the difference between calculated and simulated results are less than $1 \mathrm{~dB}$ at least for $\theta$ larger than $20^{\circ}$. Consequently, this method can be used for the functions as

$$
N(\rho)=\left\{\begin{array}{cc}
N_{1}, & 0<\rho \leq l_{1}-m_{1} \lambda / 2 \\
\left(2 n_{1}+1\right) N_{1}, & l_{1}-m_{1} \lambda / 2<\rho \leq l_{1} \\
N_{1}, & l_{1}<\rho \leq l_{2}-m_{2} \lambda / 2 \\
\left(2 n_{2}+1\right) N_{1}, & l_{2}-m_{2} \lambda / 2<\rho \leq l_{2} \\
\vdots & \vdots \\
N_{1}, & l_{k-1}<\rho \leq l_{k}-m_{k} \lambda / 2 \\
\left(2 n_{k}+1\right) N_{1}, & l_{k}-m_{k} \lambda / 2<\rho \leq l_{k} \\
N_{1}, & l_{k}<\rho \leq a
\end{array}\right.
$$

where $n_{i}$ and $m_{i}(i=1,2,3, \quad)$ are integers and $a$ is the radius of the ground screen. $N(\rho)$ is illustrated in Fig. 7 for the optimum radial wires for the angles $75^{\circ}, 65^{\circ}, 55^{\circ}, 45^{\circ}$, $35^{\circ}$ and $25^{\circ}$ respectively as well as the practical suggestions associated with (15). The suggestions indicate that $N(\rho)$ equals 73 in half domain and in the other half it is 219 which means $N_{1}=73$ and $n_{i}=1$ for all the structures. To keep the total wire lengths constant $N_{1}$ should have been assumed to be 75 , while it makes $l_{\mathrm{gs}}=7500$ as we had for the constant $N(\rho)=150$. The reduction of $N_{1}$ is to compensate the additional wires for the meandered lines as can be seen in Fig. 5.

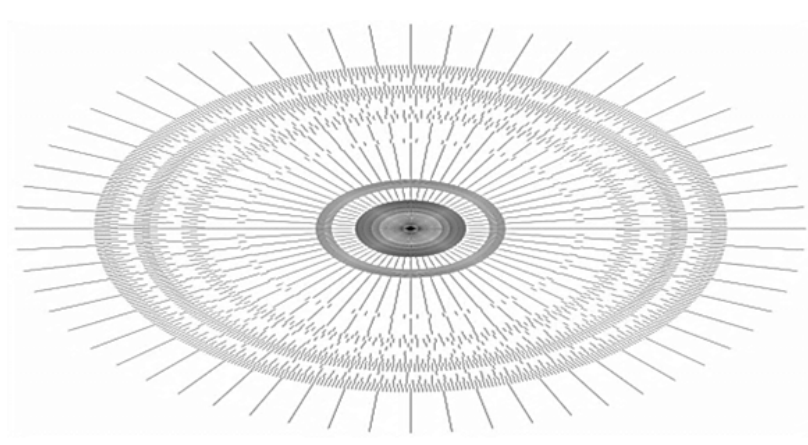

Fig. 4. Schematic of the radial wires structure optimized for $45^{\circ}$

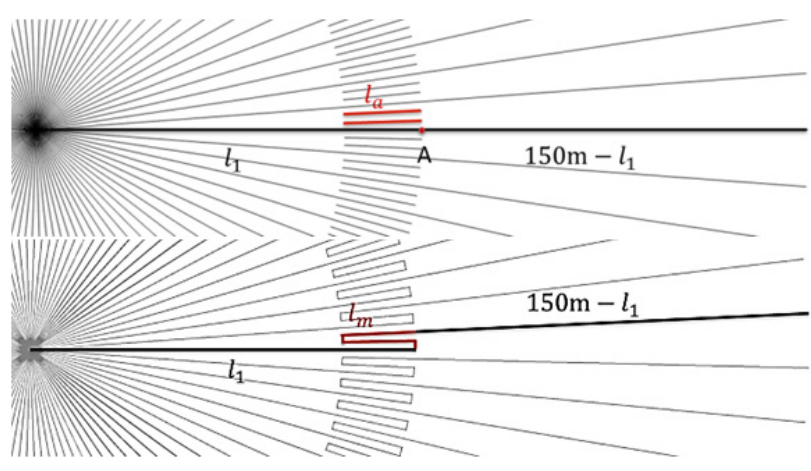

Fig. 5. Theoretical representation of (14) with the proposed practical substitution. 

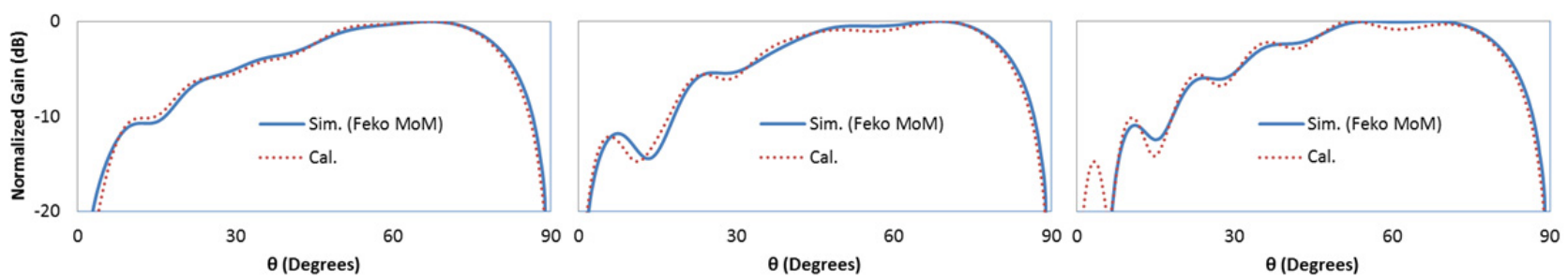

Fig. 6. Radiation patterns of the antenna on radial wires with $N(\rho)$ defined in (14) and $l_{1}=60,105,150 \mathrm{~m}$, respectively.

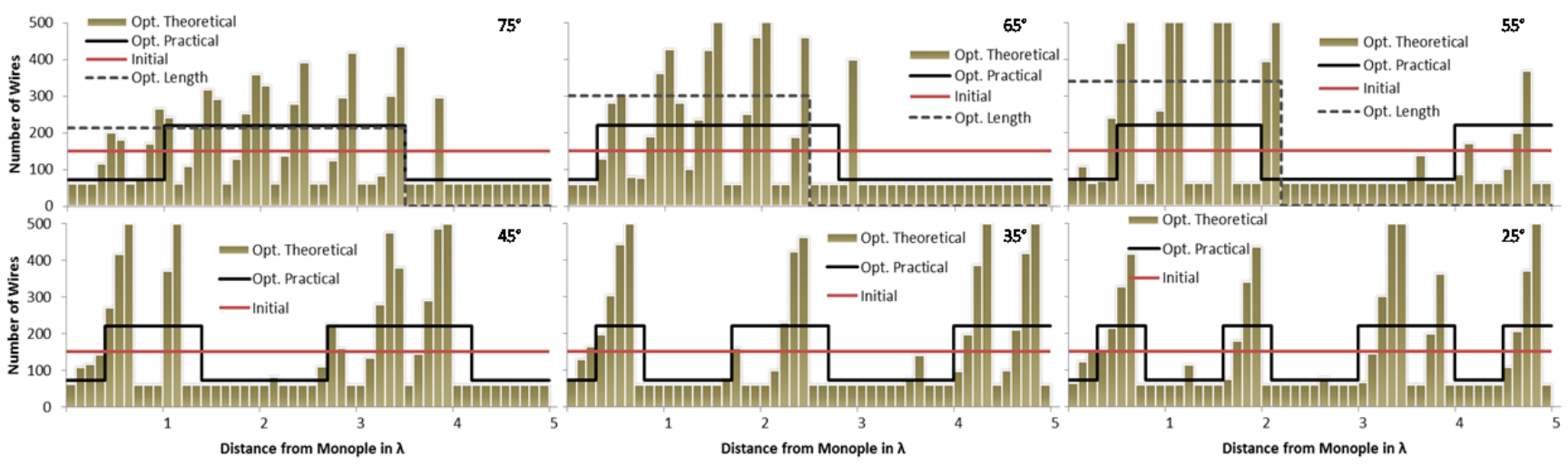

Fig. 7. Optimum number of radials for the angles $75^{\circ}, 65^{\circ}, 55^{\circ}, 45^{\circ}, 35^{\circ}$ and $25^{\circ}$ respectively and practical suggestions for each $N(\rho)$.

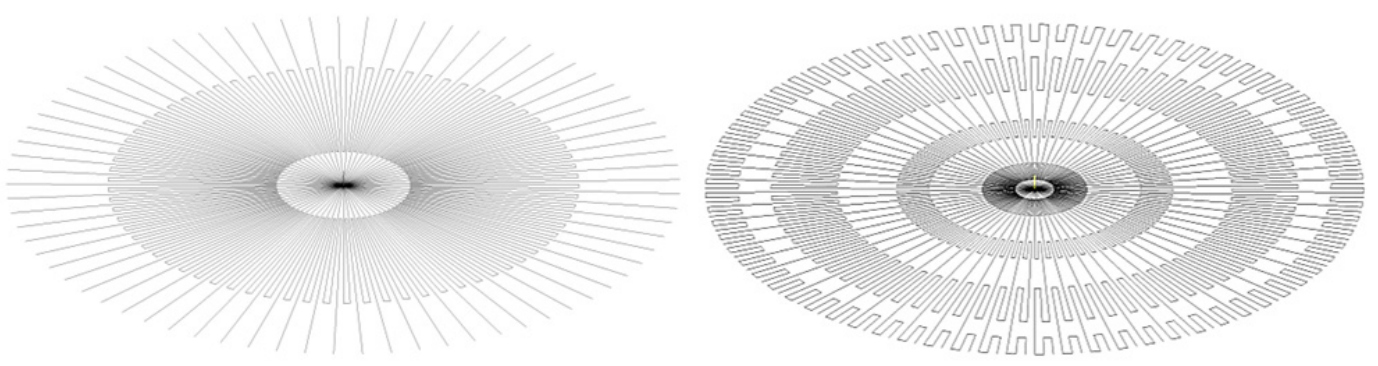

Fig. 8. Schematic of the practical radial wires structures for the beam angles $75^{\circ}$ and $25^{\circ}$.
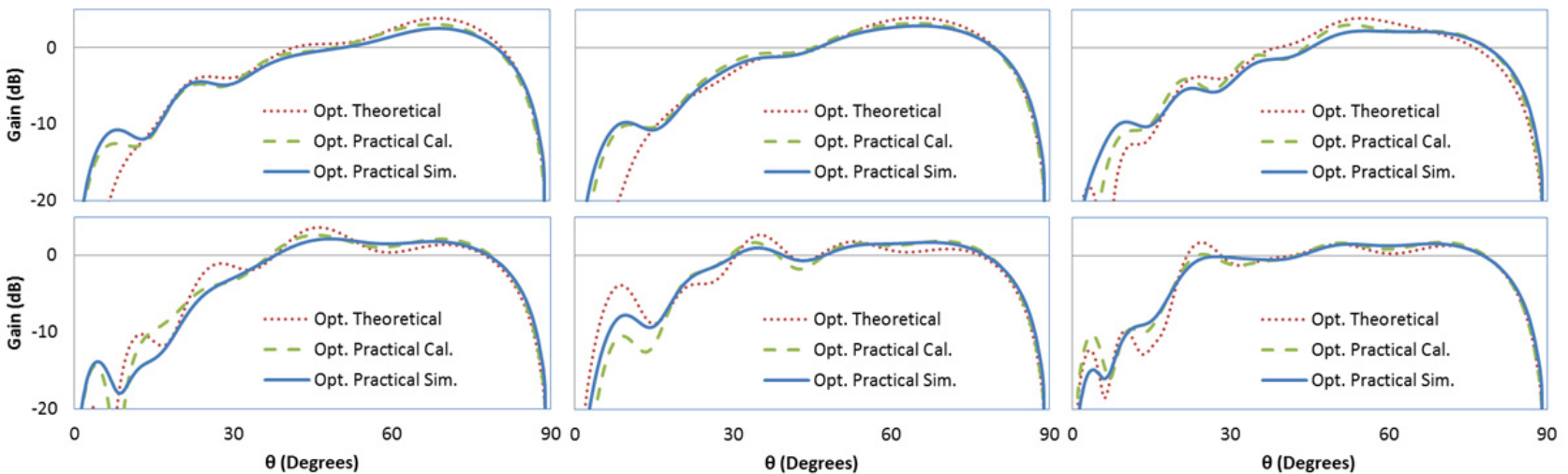

Fig. 9. Calculated radiation patterns of the antenna with theoretical and practical radial wires structures optimized for the maximum beam angles of $75^{\circ}, 65^{\circ}, 55^{\circ}, 45^{\circ}, 35^{\circ}$ and $25^{\circ}$ respectively. The Calculated results of the antenna with practical radial wires are validated by FEKO simulation results.

In addition, it can be observed that for the structures with beam angles of $75^{\circ}, 65^{\circ}, 55^{\circ}, 45^{\circ}, 35^{\circ}$ and $25^{\circ}$ we have $k=1, k=1, k=2, k=2, k=3$ and $k=4$, respectively and for $55^{\circ}, 35^{\circ}$ and $25^{\circ}, l_{k}$ equals $a$. The schematics of the practical ground screens with the functions shown in Fig. 7 are represented in Fig. 8 with meandered lines for the beam angles $75^{\circ}$ and $25^{\circ}$. The radiation patterns of theoretical and practical radial wires structures for the given beam angles are provided in Fig. 9 and the practical results are validated with the simulation of the meandered structures using MoM in FEKO. Radiation patterns of the structure with a beam angle of $45^{\circ}$ are indicated in Fig. 10 at $9 \mathrm{MHz}$, 


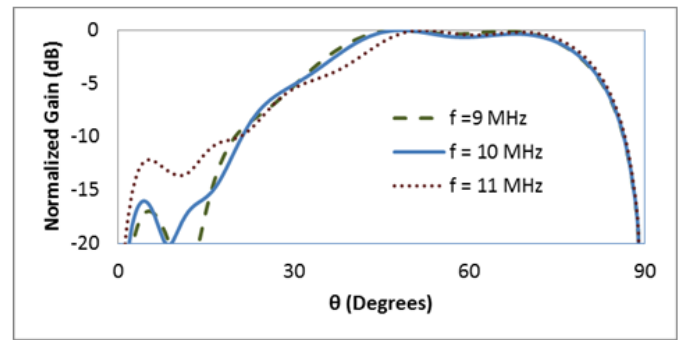

Fig. 10. Simulated radiation patterns of the antenna with beam angle of $45^{\circ}$ at $9 \mathrm{MHz}, 10 \mathrm{MHz}$ and $11 \mathrm{MHz}$.

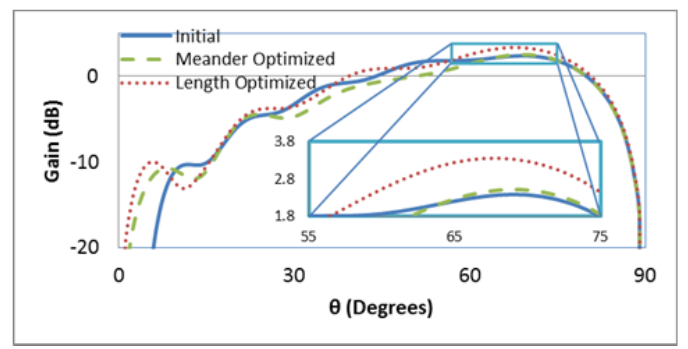

(a)

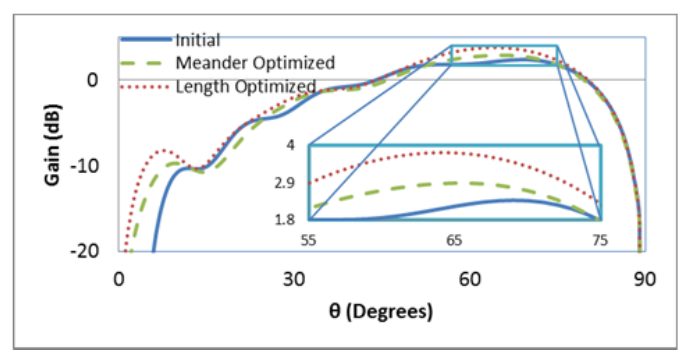

(b)

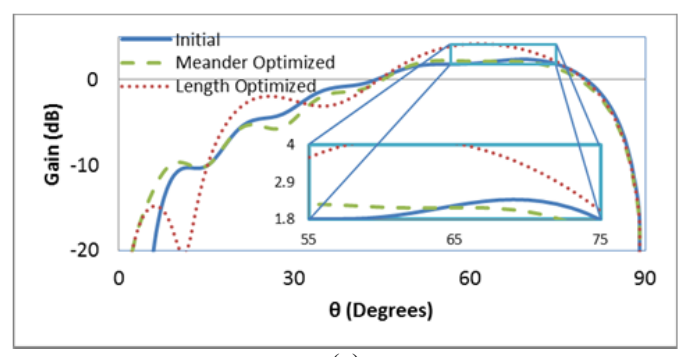

(c)

Fig. 11. Comparison between simulated radiation patterns of the antennas with 150 radial wires, practically optimized radial wires and length optimized radial wires for the beam angles of a) $75^{\circ}$, b) $65^{\circ}$ and c) $55^{\circ}$.

$10 \mathrm{MHz}$ and $11 \mathrm{MHz}$ as an example to indicate the frequency dependency. It can be observed that the difference in direction of maximum gain is about $5^{\circ}$ at these frequencies for this structure.

Nevertheless, some limitations can be observed for practical implementation of low and high angle structures (high angles correspond to small values of $\theta$ and vice versa). For the high angle structure with the desired beam direction at $\theta=25^{\circ}$, there is a local maximum on this angle and the gains at $\theta=45^{\circ}$ to $80^{\circ}$ are higher. Anyhow, it can be observed that the gain of this structure at $\theta=25^{\circ}$ is higher than other angles. Also for low angle structures we can see that the beam angle cannot exceed $\theta=70^{\circ}$. More- over it can be observed from Fig. 11(a) that the improvement of the gain is not significant compared with that of the initial structure with constant $N(\rho)=150$. It could be predicted from the first diagram of Fig. 7 while the theoretical optimum function is widely distributed and we cannot find the practical structure as other angles.

The best solution for low angle radiation is to choose an appropriate screen size. It can be recognized from Fig. 7 that the wires after $\rho=105 \mathrm{~m}$ are trivial. Therefore it is shown in Fig. 11(a) that the radiation pattern of the antenna with 214 radial wires of $105 \mathrm{~m}$ gives the best response at low angles keeping $l_{\mathrm{gs}}$ unchanged. Also for $65^{\circ}, 300$ radial wires of $75 \mathrm{~m}$ and for $55^{\circ}, 340$ radial wires of $66 \mathrm{~m}$ are the best as shown in Fig. 11(b) and (c). It can be seen in Fig. 11(c) that the beam angle cannot be reduced below $60^{\circ}$. Consequently, the initial radial wires with optimized length or the ground screen size are the best for low angle radiation and the meandered lines can be used for higher angles.

\section{Conclusion}

The optimum functions for numbers of radial wires of a quarter wave monopole for arbitrary elevation angles between $\theta=25^{\circ}$ to $\theta=75^{\circ}$ with the ground screen radius of $5 \lambda$ are presented in this paper. We considered HF band for our simulations without loss of generality, however the results can be applied for all radio frequencies. It is indicated that for low elevation angle radiation simple radial wires are the best practical solution and the ground screen size should be optimized. For higher elevation angles between $\theta=25^{\circ}$ to $\theta=45^{\circ}$ the proposed meandered lines are the best solutions. Also the proposed method cannot be used for very high elevation angles since the gain of a monopole is indeed low at these angles.

\section{References}

[1] TRAINOTTI, V., DORADO, L. A. Short low- and mediumfrequency antenna performance. IEEE Antennas and Propagation Magazine, 2005, vol. 47, no. 5, p. 66-90. ISSN: 1045-9243, DOI: 10.1109/map.2005.1599168

[2] WAIT, J. R. Antenna Performance Influenced by the Finite Extent and Conductivity of Ground Planes: A Collection of Reprints. No. M90-79. MITRE CORP BEDFORD MA, 1990.

[3] NORTON, K. A. The propagation of radio waves over the surface of the Earth and in the upper atmosphere. Proceedings of the Institute of Radio Engineers, 1937, vol. 25, no. 9, p. 1203-1236. ISSN: 0096-8390, DOI: 10.1109/JRPROC.1937.228544

[4] JOHNSON, R.C., JASIK, H. Antenna Engineering Handbook. 2nd ed. New York (USA): McGraw Hill, 1984. ISBN: 9780070323810.

[5] GAILEY, P.C. Modeling and Measurement of Electromagnetic Fields Near LORAN-C and OMEGA Stations. Philadelphia (PA, USA): EC Corp., Oak Ridge, TN. 1987.

[6] MILlER, E. K., DEADRICK, F. J., HENRY, W. O. Computer evaluation of large low-frequency antennas. IEEE Transactions on Antennas and Propagation, 1973, vol. 21, no. 3, p. 386-389. DOI: 10.1109/tap.1973.1140513 
[7] FARTOOKZADEH, M., MOHSENI ARMAKI, S.H., RAZAVI, S.M.J., RASHED-MOHASSEL, J. Fast method for the calculation of radiation resistance of a catenary element antenna applied to the optimization of a Loran transmitter antenna and a scaled model fabrication. Journal of Electromagnetic Waves Applications, 2014, vol. 28, no. 9, p. 1044-1055. ISSN: 0920-5071, DOI: $10.1080 / 09205071.2014 .904757$

[8] FARTOOKZADEH, M., MOHSENI ARMAKI, S.H., RAZAVI, S.M.J., RASHED-MOHASSEL, J. Modification of square top loaded low frequency antennas with investigations on catenary networks analysis. Journal of Electromagnetic Waves and Applications, 2015 , vol. 29 , no. 1 , p. 92-103. ISSN: 0920-5071, DOI: $10.1080 / 09205071.2014 .981643$

[9] WEINER, M. M. Monopole Antennas. New York-Basel: Marcel Dekker Inc., 2003. ISBN: 0824748441

[10] TRETYAKOV, S. Analytical Modeling in Applied Electromagnetics. Boston: Artech House, 2003. ISBN: 1580533671

[11] WAIT, J. R. The ancient and modern history of EM ground-wave propagation. IEEE Antennas and Propagation Magazine, 1998, vol. 40, no. 5, p. 7-24. DOI: 10.1109/74.735961

[12] DORADO, L. A., TRAINOTTI, V. Simplified calculation of ground losses in low-and medium-frequency antenna systems. IEEE Antennas and Propagation Magazine, 2006, vol. 48, no. 6, p. 70-81. DOI: 10.1109/map.2006.323345

[13] RAFUSE, R. P., RUZE, J. Low-angle radiation from vertically polarized antennas over radially heterogeneous flat ground. Radio Science, 1975 , vol. 10 , no. 12, p. 1011-1018. ISSN: 0048-6604, DOI: $10.1029 / \mathrm{rs} 010 \mathrm{i} 012 \mathrm{p} 01011$

[14] COLEMAN, C. J. The effect of an imperfectly conducting plane upon an incident electromagnetic field. IEEE Transactions on Antennas and Propagation, 1997, vol. 45, no. 10, p. 1445-1450. DOI: $10.1109 / 8.633848$

[15] WEINER, M. M., ZAMOSCIANYK, S., BURKE, G. J. Radiation efficiency and input impedance of monopole elements with radialwire ground planes in proximity to Earth. Electronics Letters, 1992, vol. 28 , no. 16 , p. 1550-1551. ISSN: 0013-5194, DOI: 10.1049/el:19920984

[16] BURKE, G. J., POGGIO, A. J. Numerical Electromagnetics Code (NEC)-Method of Moments. Technical Document 116, Naval Ocean Systems Center, San Diego, CA, January 1981. DOI: 10.1109/aps.2004.1331976

[17] FEKO User Manual. EMSS-EM Software and Systems. Stellenbosch, South Africa, 2000.

[18] WAIT, J. R., WEINER, M. M. Comment: Radiation efficiency and input impedance of monopole elements with radial-wire ground planes in proximity to Earth (and reply). Electronics Letters, 1992, vol. 28 , no. 25 , p. 2329-2329. ISSN: 1350-911X, DOI: 10.1049/el:19921499

[19] TENG, C., KING, R. J. Surface fields and radiation patterns of a vertical electric dipole over a radial-wire ground system. Electromagnetics, 1981, vol. 1, no. 1, p. 101-116. DOI: $10.1080 / 02726348108915127$

[20] JIN, Y.-S., ADACHI, S., SAWAYA, K. Monopole antenna on radial-wires over flat earth. Journal of Electromagnetic Waves and Applications, 1990, vol. 4, no. 1, p. 49-59. DOI: $10.1163 / 156939390 \times 00447$
[21] ABBOTT, F. Design of optimum buried-conductor RF ground system. Proceedings of the Institute of Radio Engineers, 1952, vol. 40, no. 7, p. 846-852. DOI: 10.1109/jrproc.1952.273852

[22] MACFARLANE, G. G. Surface impedance of an infinite parallelwire grid at oblique angles of incidence. Journal of the Institution of Electrical Engineers - Part IIIA: Radiolocation, 1946, vol. 93, no. 10 , p. $1523-1527$. DOI: $10.1049 /$ ji-3a-1.1946.0256

[23] GREEN, H. E. Derivation of the Norton surface wave using the compensation theorem. IEEE Antennas and Propagation Magazine, 2007, vol. 49, no. 6, p. 47-57. DOI: 10.1109/map.2007.4455846

[24] LIPSCHUTZ, S., SPIEGEL, M., LIU, J. Schaum's Mathematical Handbook of Formulas and Tables. McGraw-Hill Osborne Media, 1999. ISBN: 0071795375

[25] BORN, M., WOLF, E. Principles of Optics. London: Pergamon Press, 2000. ISBN: 0521784492

\section{About the Authors ...}

Mahdi FARTOOKZADEH was born in Tehran. He received his B.Sc. from the University of Tehran in 2009. He is currently working toward the Ph.D. degree in Malek Ashtar University. His main research interests are radio wave propagation, antennas and microwave networks.

Seyyed Hossein MOHSENI ARMAKI was born in Kashan, Iran. His received the M.Sc. in 1995 and Ph.D. in 2011 from KNTU. His research interests include antenna, antenna measurement and electromagnetics wave.

Sayed Mohammad Javad RAZAVI was born in Kashan, Iran in 1976. He received the B.Sc. degree from Isfahan University of Technology (IUT), M.Sc. degree from Malek Ashtar University of Technology (MUT), Tehran, and Ph.D. degree from Iran University of Science and Technology (IUST), all in Electrical Engineering in 1999, 2002, and 2009, respectively. Currently, he is a researcher in EEUC/Electrical \&Electronic University complex/MUT Tehran, Iran.

Jalil RASHED-MOHASSEL was born in Birjand, Iran and received his Ph.D. degree in Electrical Engineering from University of Michigan, Ann Arbor in 1982. His research interests include antennas, EM theory and EMC/EMI. In 1994 he joined the University of Tehran where he is doing teaching and research as a professor in the school of ECE. He is the author of three books and has published more than 200 papers in refereed journals and conference proceedings. He is a principal member of the Center of Excellence on Applied Electromagnetic Systems (CEAES), and the director of the microwave laboratory. He is a distinguished professor and the recipient of several national prizes. 